ENCYCLOPEDDIE Encyclopédie berbère

BERBERE

$25 \mid 2003$

25 | Iseqqemâren - Juba

\title{
Iwellemmeden Kel Ataram
}

H. Claudot-Hawad

\section{OpenEdition}

Journals

Édition électronique

URL : http://journals.openedition.org/encyclopedieberbere/1458

DOI : 10.4000/encyclopedieberbere.1458

ISSN : 2262-7197

\section{Éditeur}

Peeters Publishers

\section{Édition imprimée}

Date de publication : 1 septembre 2003

Pagination : 3822-3828

ISBN : 2-7449-0424-4

ISSN : 1015-7344

\section{Référence électronique}

H. Claudot-Hawad, «Iwellemmeden Kel Ataram », Encyclopédie berbère [En ligne], 25 | 2003, document

176b, mis en ligne le 01 juin 2011, consulté le 24 septembre 2020. URL : http://

journals.openedition.org/encyclopedieberbere/1458; DOI : https://doi.org/10.4000/

encyclopedieberbere.1458

Ce document a été généré automatiquement le 24 septembre 2020.

(c) Tous droits réservés 


\title{
Iwellemmeden Kel Ataram
}

\author{
H. Claudot-Hawad
}

1 Les Iwellemmeden de la région de Menaka sont appelés Kel Ataram, «Ceux de l'Ouest », pour les distinguer des Iwellemmeden Kel Denneg, «Ceux de l'Est ». Ces derniers, à la suite d'un conflit politique, se seraient séparés de la maison-mère au XVIII siècle ou au tout début du XIX ${ }^{e}$ siècle selon les sources (Richer 1924), scission interprétée également comme un processus long qui s'amorce dès le XVII ${ }^{e}$ siècle et s'achève au début du XIX $\mathrm{X}^{\mathrm{e}}$ siècle (Brock 1983).

Depuis les indépendances africaines dans les années 1960, les deux formations politiques, réduites après la colonisation à des entités «tribales" restreintes, sont séparées par une frontière étatique qui ne correspond pas d'ailleurs aux anciennes limites territoriales : ainsi, le centre administratif local dont relèvent les Kel Ataram est Menaka situé dans l'actuel Mali, tandis que les Kel Denneg sont rattachés à Tahoua qui fait partie du Niger.

\section{Émergence d'une formation politique hégémonique}

3 L'étude historique la plus fouillée sur les Iwellemmeden Kel Ataram reste pour l'instant celle d'un médecin des troupes coloniales, le Dr Richer (1924) qui s'appuie notamment sur des sources locales orales (en touareg et en arabe) et écrites (manuscrits du $\mathrm{XVI}^{\mathrm{e}}$, $\mathrm{XVII}^{\mathrm{e}}$ et $\mathrm{XVIII}{ }^{\mathrm{e}}$ siècles en arabe), ainsi que sur les archives des postes militaires français installés au cours de la conquête de cette région. Selon cet auteur, dont les données et souvent les interprétations sont reprises par la plupart des études postérieures, c'est en 1800 qu'une branche de la confédération guerrière des Iwellemmeden aurait contesté le pouvoir de l'amenukal Kawa, placé à la tête d'un immense ensemble politique qui englobait toute la région du fleuve Niger, de Tombouctou à Say. Le parti d'opposition, regroupant plusieurs tribus, aurait été vaincu et la coalition se serait retirée vers l'est en s'autonomisant et en s'appuyant sur de nouvelles alliances.

4 De leur côté, les Iwellemmeden Kel Denneg situent la scission avec les Kel Ataram à la fin du XVII siècle. L. Brock (1983) souligne que les deux versions, dans leur aspect 
contradictoire, renvoient précisément au fait que la rupture ne s'est pas faite d'un coup, mais s'est déroulée sur un siècle environ. Son argumentation s'appuie sur l'analyse des jeux d'alliances et d'oppositions politiques qui se sont dessinées durant cette période au sein du pôle politique Iwellemmeden.

5 Avant cet épisode de scission, Richer situe l'émergence des Iwellemmeden à la fin du $\mathrm{XVI}^{\mathrm{e}}$ siècle dans l'Adagh* où ils entrent en compétition avec la confédération existante des Kel Tademekkat, tandis que les groupements les plus anciens qui peuplaient l'Adagh auparavant comme les Idnan, Imededaghen et Udalen, seraient partis vers le sud entre le $\mathrm{x}^{\mathrm{e}}$ et le $\mathrm{XIII}{ }^{\mathrm{e}}$ siècle. Vers 1653 , selon Richer, les Iwellemmeden auraient vaincu les Kel Tademekkat et les auraient repoussés hors de l'Adagh, en direction du sud. Quarante ans plus tard, sous le règne de Karidenna vers 1690, les Iwellemmeden seraient à leur tour descendus vers le fleuve, installant alors leur hégémonie sur toute la région.

6 Lhote (1955) conteste cette reconstruction historique en relevant notamment que Tombouctou fut attaquée par les Iwellemmeden dès 1647 (Târîkh as-Soûdân). Selon cet auteur, «ce sont en réalité les Iwellemmeden qui durent quitter l'Adrar les premiers sous la poussée des Kel-Tademekkat». Plus tard, avec la montée en puissance des Iwellemmeden, les Kel Tademekkat auraient préféré abandonner l'Adagh pour se rendre dans la région de Tombouctou: dans ce but, ils auraient dépêché en 1655, d'après le Târîkh as-Soûdân, une délégation auprès du pacha Mohamed ben Ahmed pour lui demander de s'installer dans le voisinage de la ville.

7 Quelles que soient les hypothèses, l'histoire de la confédération des Iwellemmeden s'inscrit localement dans le cadre des rapports de force établis avec plusieurs groupes de pression, en particulier la formation politique antérieure des Touaregs Kel Tademekkat, les Marocains qui s'installent à la fin du $\mathrm{xvI}^{\mathrm{e}}$ siècle dans la région de Tombouctou puis de Gao, les sédentaires Songhay et les Maures. À la fin du XVIII siècle, les Iwellemmeden dominent toute la région du fleuve. Leur expansion politique se poursuit en incluant dans leur protectorat des groupements arabophones Kounta et Berabisch au nord et Rhattafen au sud ainsi que des Peuls. Cette période, jusqu'au début du XIX ${ }^{e}$ siècle, représente l'apogée du pouvoir des Iwellemmeden, qui doivent ensuite se confronter aux Peuls du Macina qui s'emparent de Tombouctou en 1826, puis lutter au nord contre la pression de plus en plus affirmée des Kel Ahaggar qui leur disputent l'Adagh, et à l'est contre celle des Kel Ayr. Des tendances autonomistes se manifestent chez certains groupements clients qui privilégient de nouveaux alliés.

\section{Organisation sociale}

8 Au début du xx ${ }^{e}$ siècle, la confédération des Iwellemmeden Kel Ataram contrôle encore un territoire immense, qui s'étend sur tout l'ouest du pays touareg à partir de la ligne Kidal-Menaka jusqu'au lac Faguibine. Ce vaste pôle politique, véritable empire, est désigné tantôt sous le nom de ses dirigeants Iwellemmeden, tantôt (notamment par les Touaregs de l'Est) sous le nom de Tademmekat élargi à l'ensemble de la formation politique. Celle-ci est bâtie sur le mode confédéral. Sa structure réserve à chaque unité articulée à l'ensemble une certaine autonomie et autorise ainsi la coexistence en son sein de modèles différents d'administration des hommes et des biens. Aux clivages 
politiques se superposent des spécialisations économiques et souvent aussi des disparités linguistiques, sociales ou culturelles.

9 Ainsi, les nobles Iwellemmeden, guerriers éleveurs de chameaux et de chevaux, font partie des « gens du j », c'est-à-dire parlent la tamajaq, l'une des variantes de la langue touarègue pratiquée en majorité dans l'Aïr. Ils sont organisés en lignages patrilinéaires et définissent selon cette même voie dite « du dos » (aruri) les règles sociales telles que la succession et la transmission des droits, l'héritage, la résidence... Par contre, leurs clients ou leurs dépendants touaregs, qu'ils soient de la rive nord ou sud du fleuve (Awza et Gourma), ou de l'Adagh au nord, parlent la tamašaq.

De même, le système de filiation et les règles de transmission des biens et des pouvoirs varient selon les groupements. Si les nobles Iwellemmeden sont patrilinéaires, par contre certains de leurs clients comme les Imededaghen, guerriers et grands éleveurs de zébus et de chevaux, ainsi que les tributaires de ces derniers qui s'occupent essentiellement de petit bétail, forment en majorité des unités matrilinéaires descendant d'une ancêtre féminine, et privilégient dans leur organisation sociale la matrilinéarité ou voie « du ventre ».

11 Entre ces deux extrêmes, les nobles du Gourma comme par exemple les Igawdaren à l'est ou les Tengeregef à l'ouest dans la région de Tombouctou - dont certains, installés au XVIII ${ }^{e}$ siècle sur les rives du fleuve, se disent apparentés aux Iwellemmeden - parlent la tamašaq, mais sont organisés patrilinéairement ; notons que leurs dépendants directs suivent les mêmes règles. La conservation et l'épanouissement de ces particularités, malgré l'intensité et la fréquence des relations entre groupements, contraste avec le laminage des diversités culturelles observable dans les sociétés à pouvoir centralisé.

12 La structuration hiérarchique de ce grand pôle politique de l'ouest sous l'autorité des Iwellemmeden apparaît complexe. Les divers niveaux d'organisation politique où se distribuent les unités sociales variées sont souvent dédoublés par rapport aux formations politiques touarègues voisines. Ainsi, au début du $\mathrm{xx}^{\mathrm{e}}$ siècle, quatre échelons hiérarchisés se dessinent. Au sommet, dominent les Iwellemmeden Kel Ataram, puis la noblesse issue de l'ancien pôle indépendant de la Tademekkat, comme les Igawdaren et les Iheyawen-n-Alad installés dans le Gourma, ou les Tengeregef dans la région de Tombouctou, puis les confédérations clientes et guerrières comme par exemple les Imededaghen, enfin les « pauvres » ou les protégés. Même s'il existe de multiples façons de faire comprendre clairement la position d'un groupement dans cette classification à degrés multiples, les catégories linguistiques s'appliquant au statut politique proprement dit se limitent à deux termes : imuša (suzerains) qui s'applique aux deux premiers niveaux (Iwellemmeden comme Igawdaren, Tengeregef...), tandis que imyad (tributaires) peut servir à définir à la fois les suzerains d'une confédération cliente et leurs dépendants. Ces derniers cependant sont appelés plus souvent tilaqawin, «les pauvres ». C'est pourquoi, contrairement à l'Ahaggar, l'Ajjer ou l'Aïr, où le terme imyad manque de gloire et ne s'applique qu'aux tribus reconnues historiquement dépendantes, dans le Gourma, ce mot avait, du moins dans les années 1980 lors de mes enquêtes, une connotation de noblesse et de puissance. Ce statut s'appliquait en effet à des tribus guerrières qui, tout en reconnaissant l'autorité des imuša ("suzerains", sous-entendu ici Iwellemmeden), avaient elles-mêmes sous leur domination des tribus de " pauvres" (tilaqawin). Pour les distinguer de ces derniers groupements, les rapports coloniaux les dénommaient d'ailleurs : «imyad guerriers » ou encore «imyad de grande tente ». 
13 Ces échelons qui en dépit de leur incontournable réalité demeurent informels, soulignent le caractère fluide de la hiérarchie sociale et son rééquilibrage constant en fonction de l'expansion ou de l'affaiblissement des alliances politiques. Ils s'observent également dans le reste du pays touareg, bien que dans une extension moindre (c'est pour traduire une opposition de cette nature, par exemple, que Foucauld utilise au sujet de l'Ahaggar l'expression de "suzerains de second ordre " par opposition aux suzerains dont le pouvoir prédomine).

\section{L'ordre colonial}

14 L'intrusion coloniale va détruire le pôle politique des Iwellemmeden Kel Ataram et bouleverser irréversiblement l'ordre régional. En 1894, l'armée française après plusieurs affrontements violents avec les Touaregs de la région de Tombouctou et de la partie centrale du fleuve, occupe Tombouctou. Vaincus, les Tengeregef, Iregenaten et Kel Temulayt demandent la paix. En 1896, le Lieutenant Hourst qui estime alors la force armée des Iwellemmeden à 20000 ou 30000 combattants, va signer un accord éphémère de non agression mutuelle avec le chef des Iwellemmeden, Madidou. Mais les accrochages et les attaques contre les forces d'occupation françaises se multiplient, menés par divers groupes dissidents (Kel Ahaggar, Kountas, Igawdaren, Kel Intessar, Kel Temulayt...) auxquels s'adjoignent en 1897 deux fractions des Iwellemmeden (Kel Ara et Kel Eguedesh, Richer: 143). L'occupation militaire du fleuve planifiée par les Français est engagée en 1898. Le harcèlement des tribus touarègues qui résistent entraîne de nombreuses pertes en troupeaux et en hommes. Les groupements soumis sont cantonnés sur la rive sud du fleuve (Gourma) pour les soustraire à l'influence des Iwellemmeden, repliés vers l'intérieur du pays à l'est, et qui mènent de nombreuses offensives sur le fleuve. Pour accélérer l'occupation militaire de la région Iwellemmeden, l'armée coloniale cherche des auxiliaires parmi la population, sédentaire ou nomade, qui s'est rendue et exerce de nombreuses représailles contre ceux qui refusent de collaborer (exécutions, déportations, emprisonnements, amendes, destruction de biens, interdiction des zones agricoles et des marchés, blocus...). Les Kountas sous la direction de Hamoadi, armés de fusils à tir rapide par les Français, sont utilisés pour soumettre les Iwellemmeden qui subissent de très lourdes pertes. Des pourparlers sont entamés à Gao en 1902. Laoueï (Lawey), successeur de Madidou mort trois ans plus tôt, offre de faire sa soumission «à condition d'avoir comme terrain de parcours tout le terrain entre Gao et Tombouctou " (Richer: 181). C'est finalement Firhoun (Firhun), élu amenukal à sa place, qui signera l'acte de soumission à Gao le 23 janvier 1903, « au nom des sept principales tribus nobles : Kel Koumer, Kel Tiguiouelt, Kel Ara, Ibaouanes, Tahabanaten, Iouaten, Irohorniten " (Richer : 183). Les conditions imposées ruinent l'assise territoriale, politique et économique des Iwellemmeden, en leur interdisant notamment le Gourma et l'Adrar, en leur ôtant tout droit de gestion et d'intervention sur leur ancien domaine politique, en leur imposant un lourd tribut. En 1908, dans un climat de tension permanent, est mise en place une administration directe. Comme le précise sans ambiguité Richer (p. 257) : « son aboutissement était la constitution des tribus en organismes autonomes [...], sa conclusion, finalement, était la suppression de l'Aménokal ». En novembre 1914, après une esquisse de soulèvement, Firhun est arrêté avec son entourage et condamné à dix ans de prison. Au moment où sa grâce présidentielle arrive en février 1916, le détenu vient de s'évader de Gao. Averti des projets d'insurrection qui se préparent à l'est autour de Kawsan originaire de l'Aïr, 
Ingedazen et Amud de l'Ajjer (ce dernier attaquera Djanet début mars), Firhun proclame la révolte générale. Il écrit à Musa ag Amastan, l'amenukal de l'Ahaggar sous tutelle française, pour l'inviter au soulèvement, mais ce dernier transmet la lettre aux autorités coloniales. Les colonnes françaises avec leurs auxiliaires Chaamba et Kel Ahaggar attaquent le campement des insurgés à Anderamboukan le 9 mai 1916: plusieurs centaines de guerriers Iwellemmeden périssent, ainsi que des femmes et des enfants. Firhun qui a réussi à s'enfuir sera tué deux mois plus tard au nord-est de Ménaka par des auxiliaires Kel Ahaggar. Les Kounta pillent les rescapés, continuant « à s'enrichir des dépouilles des Oulliminden » (Richer, 289). La révolte des Iwellemmeden est anéantie et celles des Touaregs de la Boucle du Niger et des sédentaires de la région de Gao sont à leur tour définitivement réprimées. À Gao, le $1^{\text {er }}$ septembre 1916, un parent de Firhun, Akorakor, est nommé amenukal des Iwellemmeden. Des sanctions sévères sont imposées et d'autres meneurs du soulèvement sont arrêtés ou tués. En décembre, c'est l'Aïr qui devient le foyer d'un soulèvement touareg général, débutant avec l'occupation d'Agadez par les troupes armées de Kawsan ag Kedda, arrivé de l'exil. Les Iwellemmeden Kel Ataram, ruinés, amoindris, détruits, ne pourront participer à cette ultime tentative touarègue contre l'occupation coloniale.

\section{Représentations des origines}

15 L'histoire orale de l'ouest du pays touareg est moins connue que celle du nord ou de l'est. Peu de travaux publiés en font mention (Barth, Norris, Richer). Les récits de fondation des Iwellemmeden, s'ils présentent des versions très différentes selon les époques et les interlocuteurs, s'organisent cependant selon un modèle classique dans l'espace touareg qui prête toujours aux dominants une origine extérieure, tandis que les dominés sont présentés comme les autochtones (Claudot-Hawad, 1982). Cet « ailleurs » varie selon les époques : ainsi, l'ancêtre fondateur des Iwellemmeden arrive suivant les récits tantôt du Yémen à l'Est, tantôt du sud marocain ou du Trarza au Nord-Ouest, tantôt de Oualata à l'Ouest... Mais l'un des traits caractéristiques de cet ancêtre fondateur par rapport à beaucoup d'autres récits touaregs, est d'abord le fait qu'il s'agit d'un homme, qui plus est étranger (arabe ou musulman). Il y a une sorte d'inversion du schéma utilisé dans les milieux matrilinéaires où l'ancêtre fondateur est une femme, représentant le cœur de la société, et qui généralement provient d'un extérieur prestigieux, se marie localement et domine, avec sa descendance, la région d'accueil. L'ancêtre masculin des Iwellemmeden arrive donc dans l'Adagh (appelé Adrar des Iforas depuis la colonisation) et à la suite de services rendus au chef de la région, à la tête des Touaregs Kel Tademmekkat, épouse la fille ou la sœur de ce dernier.

Plusieurs récits associent l'introduction de nouvelles règles et valeurs de l'ordre social, c'est-à-dire la filiation patrilinéaire et l'islam, à l'arrivée de l'ancêtre des Iwellemmeden. Au contraire, dans cette perspective, les Kel Tademekkat incarnent la continuité matrilinéaire et le refus de la religion musulmane. Cette opposition se traduit par l'appropriation de l'épopée de Koseyla*, héros de la résistance berbère contre l'islam conquérant. Le personnage de Koseyla, que les auteurs médiévaux de langue arabe décrivent le plus souvent comme un Zénète des Aurès est considéré ici comme l'ancêtre des Imededaghen matrilinéaires et appelé par le diminutif de Koseylata (Claudot-Hawad, 1985). À l'opposé, les récits de la tradition orale associent aux chefs Iwellemmeden en guerre contre les Kel Tademekkat des alliés (savant ou chef 
de guerre) qui sont des figures - souvent synthétiques comme l'a montré Norris (1975) - du monde musulman, tels Oqba ben Naffa, Yaqoub el Ansari, Sidi Oqba el Moutadjab...

17 C'est dans ce cadre que l'étymologie du terme Iwellemmeden (au singulier Awllemmed), a été rapportée à la phrase verbale : wer ilemmed, qui en touareg signifie : «il n'apprend pas ». Cette appellation renverrait donc à la position de refus des Iwellemmeden par rapport à la coutume matrilinéaire.

D'autres versions de l'histoire orale circulant plus à l'est prennent ce motif à rebours et font au contraire des Iwellemmeden des modèles de l'insoumission des nobles à l'islam et à ses valeurs prônées par les groupements religieux comme les Kel Essuk. "Ils n'apprennent pas » est pris dans le sens de "ils ne se soumettent pas ", c'est-à-dire n'acceptent aucune autorité, fût-elle divine. L'un des signes de cette insoumission pointé dans ces récits est la coiffure masculine à longues tresses que les Iwellemmeden portaient dans la première moitié $\mathrm{du} \mathrm{xx}^{\mathrm{e}}$ siècle, alors que le crâne rasé qui connote le renoncement à l'orgueil guerrier, est préféré par les ineslimen, c'est-à-dire les «musulmans » formant une catégorie sociale particulière dans l'ouest.

19 Une autre version des origines recueillie par Barth en 1854 (cité par Richer, p. 50 ; Barth 1965, p. 720) donne enfin aux Iwellemmeden un ancêtre du nom de "Siggini, originaire de la tribu des Hymiarites (gens de l'Yémen); Siggini serait l'ancêtre de Lamt, père des Lamta ». Barth fait le rapprochement entre Siggini et "Asnag, ancêtre des Sanhadja, signalé par Ibn Khaldoun». On pourrait également rapprocher la racine de Iwellemmeden de celle de Lamta (précédé de $a w$, « fils de, espèce de »), qui les resituent dans l'histoire plus large et plus ancienne du Sahara.

Certains récits recueillis dans l'Ahaggar (Claudot, 1982) mettent en scène sur le mode parental les liens des Iwellemmeden avec les autres pôles politiques touaregs. Dans ce schéma, tous sont issus de la même ancêtre, mais les Kel Ajjer, Kel Aïr, Kel Ahaggar sont présentés comme des « enfants de sœurs » ou des descendants de femmes, alors que les Iwellemmeden apparaissent comme des " enfants de frère » ou descendants d'homme, ce qui dans la logique matrilinéaire de l'Ahaggar pointe leur position et leur organisation particulières par rapport aux autres confédérations.

Enfin, selon certains points de vue défendus dans l'Aïr, les Iwellemmeden viendraient de l'Est (d'où leur parler en " $\mathrm{j}$ ») et seraient apparentés aux Ikazkazen, issus, comme eux, à l'origine des Uraghen de l'Ajjer, les replaçant ainsi dans le vaste réseau de relations complexes qui liaient les quatre grands pôles politiques touaregs avant l'intervention coloniale.

\section{BIBLIOGRAPHIE}

Sources traduites de l'arabe :

Târîkh el-Fettâch, écrit vers 1550 par Mahmoud KaTı (sur l'empire de Gao), Traduit par O. Houdas \& M. Delafosse, 1913 
Târîkh as-Soûdân, écrit vers 1650 par Abederrahman ben Abdallah ben 'Imran ben 'Amir ES-SA'DI, traduit par O. Houdas \& E. Benoit, Maisonneuve, Paris, 1980.

Tậlkîrat an-Nisiân (« Rappel de l'oubli »), achevé vers 1750, traduit par Houdas en 1901 (histoire de Tombouctou aux XVII et XVIII ${ }^{\mathrm{e}}$ siècles sous les Marocains)

Autres références

ABItBol M. (éd.), Tombouctou au milieu du XVIII siècle d'après la chronique de Mawlay al-Qasim B. Mawlay Sulayman, Maisonneuve et Larose, Paris, 1982.

ANONYME, Chez les Oulliminden, L'Afrique française 27, 1917 : 298-299.

AOUAD-BADOUAL R., « Le rôle de 'Abidin el Kounti dans la résistance nomade à la conquête française de la Boucle du Niger (1894-1902) », in Le politique dans l'histoire touarègue (H. Claudot-Hawad dir.), Cahiers de l'IREMAM (4), Aix-en-Provence, 1993, pp. 35-48.

AymARD, Capitaine A., «Les Touareg de la région de Tombouctou », in Bulletin du Comité de l'Afrique Française, $n^{\circ}$ 20, 1910, 399-401.

AYMARD, Capitaine A., Les Touareg, Paris, Hachette, 1911.

BARTH H., Travels and Discoveries in North and Central Africa, 1849-1855, 1965, Londres, Frank Cass \&

Co. LTD, 3 vol.

BÉTRIX Ct, La pénétration touareg, Paris, Lavauzelle, 1911.

Brock L., The Tamejjirt. Kinship and Social History in a Tuareg Community, Ph.D., Columbia University, New York, 1983.

CAILlÉ R., Voyage à Tombouctou, Paris, LD/La Découverte, 1985.

CAMEL F, L'administration coloniale et les Touaregs en AOF, (1914-1934), Thèse de $3^{\mathrm{e}}$ cycle en Histoire, Université de Provence, 2003.

CHAVENTRÉ A., Évolution anthropo-biologique d'une population touarègue. Les Kel Kummer et leurs apparentés, Paris, PUF, $334 \mathrm{p}$.

CISSÉ B., « Un grand résistant malien : Firhoun », Études maliennes 10, 1974, 1-8.

CLAUDOT-HAWAD H., Adagh, Encyclopédie Berbère, II, 1985.

CORTIER M., D’une rive à l'autre du Sahara (Mission Arnaud-Cortier, 15 fév.-24 juin 1907), Paris, Larose, 1908.

CORTIER M., « Le pays des Oulliminden », La Géographie n²1, 1910, pp. 221-236.

DELAFOSSE M., Haut-Sénéragl-Niger (Soudan français), Paris, Larose, 1912, 3 vol.

HOURST Lt, La mission Hourst, Paris, Pion, 1898.

LHOTE H., « Aoullimmiden (Ioullemedden) », Encyclopédie berbère, Cahiers provisoires, $\mathrm{n}^{\circ}$ 28, $1981,4 \mathrm{p}$.

MACNAMARA WINTER M., A study of family and Kinship Relations in a Pastoral Twareg Group of Northern Mali, Phd, Université de Cambridge, 1984, 222 p. [voir compte-rendu par H. Claudot-Hawad., in Annuaire de l'Afrique du Nord, t. XXIV, 1985, 784-790].

MILBURN M., « The Awelimmiden : history of a name », Sahara, 2, 1989, p. 99, 10 réf. Bibl.

NICOLAS F., Tamesna : les Iwillemeden de l'Est, Paris, Imprimerie nationale, 1950. 
NORRIS H. T., The Tuaregs. Their Islamic Legacy and its Diffusion in the Sahel, Warminster (UK), Aris and Phillipps Ltd, 1975.

NORRIS H. T., « L'amenokal K'awa ou l'histoire des Touaregs Iwellemmeden », in Les Africains, t. XI, Éd. Jeune Afrique, Paris, 1978.

RICHER Dr A., Les Touareg du Niger. Les Oullimminden, Paris, Larose, 1924.

SALIFOU A., « Les Français, Firhoun et les Kounta, 1902-1916 », Journal de la Société des Africanistes A3, 1973, 175-195.

URVoY Cpe Y, Histoire des populations du Soudan Central (Colonie du Niger), Paris, Larose, 1936.

VERMALE Dr P., Au Sahara pendant la guerre européenne, Correspondance et notes, 1914-1917, Alger, 1925 (réimprimé par Éd. Gandini, 1995)

INDEX

Mots-clés : Tribu, Touareg 